\title{
Über den Stellenwert der Duplexsonografie und der transkraniellen Doppler-Emboliedetektion bei der Moyamoya-Angiopathie
}

\section{Warum dieser Fall?}

Der Fall verdeutlicht den besonderen Stellenwert von Duplexsonografie und spezieller Dopplersonografie in $\mathrm{Zu}-$ sammenschau mit konventioneller Angiografie und MRT bei der MoyamoyaAngiopathie.

\section{Einleitung}

Die Moyamoya-Angiopathie ist eine seltene idiopathische Vasopathie, die durch progrediente beidseitige Stenosen und im Verlauf Verschlüsse der intrakraniellen Abschnitte der A. carotis interna, der proximalen Abschnitte der A. cerebri media und anterior gekennzeichnet ist. Namensgebend ist das dichte Kollateralnetz, das angiografisch mit einer Rauchwolke verglichen wurde, weshalb das japanische Wort Moyamoya (Wölkchen, Rauchschwade) gewählt wurde [1]. Genaue Daten zur Epidemiologie fehlen [2]. Es handelt sich um eine in Europa sehr seltene Erkrankung, wobei auch von einer höheren Dunkelziffer auszugehen ist. Eine japanische Studie mit 2075 Patienten mit Moyamoya-Erkrankung hatte eine jährliche Inzidenz von 0,35 und eine jährliche Prävalenz von 3,16 auf 100000 identifiziert [3]. Neuere Studien hatten Inzidenzen von 0,94 in Japan berichtet [4].

Als Moyamoya-Syndrom wird die Angiopathie dann bezeichnet, wenn andere Erkrankungen ursächlich oder assoziiert mit der Vasopathie auftreten, so z.B. eine Neurofibromatose von Recklinghausen, ein Downsyndrom oder die Erkrankung nach einer Meningitis oder Vaskulitis besteht [2].

Ätiologisch und pathophysiologisch ist die Genese der idiopathischen Moyamoya-Erkrankung unklar [5]. Die Double-HITSHypothese geht von verschiedenen Faktoren als ursächlich für die exzentrische fibrozelluläre Verdickung der Intima und die abnorm gewundene Elastica aus [2]. Eine genetische Suszeptibilität scheint eine Rolle zu spielen, wobei dies vornehmlich bei Ostasiaten mit der Foundervariante p.R4810K des RNF213-Gens auf Chromosom 17Q25.3 beschrieben ist $[6,7]$. Außerdem können endotheliale Progenitorzellen, Intimaabnormitäten

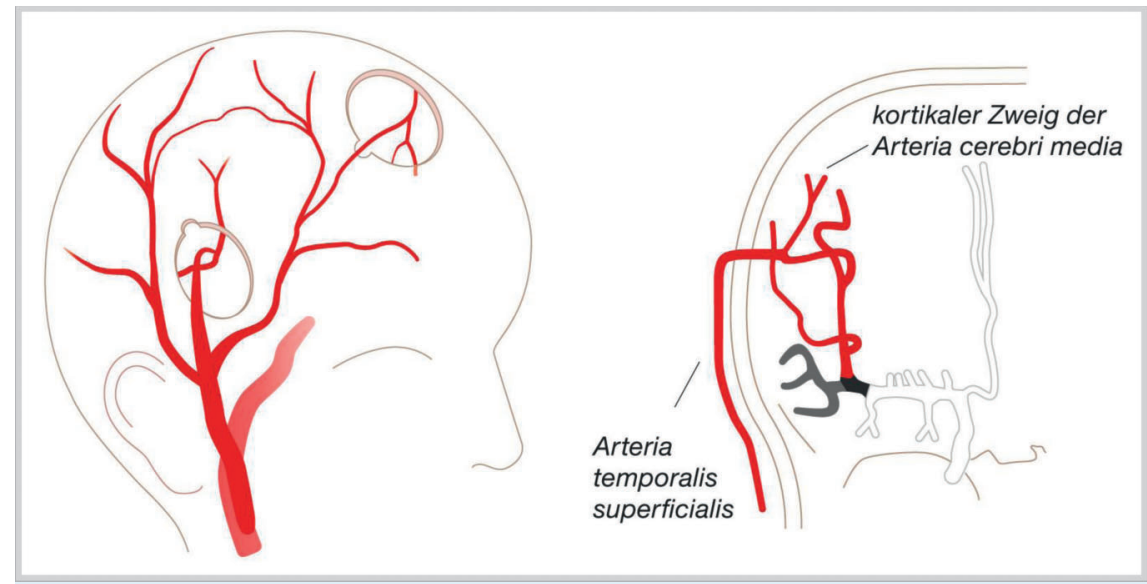

Abb.1 Prinzip einer revaskularisierenden A. temporalis superficialis - A. cerebri media (STA-MCA)Bypass-Op bei Moyamoya-Angiopathie (Bild: Krämer M, Khan N, Diesner F, Roder C. MoyamoyaErkrankung und Moyamoya-Syndrom - Informationen für Betroffene, Angehörige und Interessierte, Deutscher Medizin Verlag, Münster 2014).

und Triggerfaktoren wie Infektionen, hormonelle Faktoren, Autoimmunfaktoren sowie hämodynamischer Stress und Rezeptorabnormalität im C1-Bereich eine Rolle spielen und schließlich zu den histologischen Veränderungen Anlass geben, die zu Ischämien führen und schließlich zur Vaskulogenese über Zytokinausschüttungen [2].

Klinisch manifestiert sich die MoyamoyaErkrankung mit flüchtigen oder manifesten zerebralen Ischämien, aber auch zerebrale Blutungen und Bewegungsstörungen sowie Kopfschmerzen finden sich [8]. Therapeutisch wird weiterhin die revaskularisierende Operation mittels Bypassoperation von extrakraniellen Gefäßen zu intrakraniellen Gefäßen, insbesondere die Verbindung zwischen A. temporalis superficialis und distalem Mediaast als Erfolg versprechend angesehen [9] ( $\bullet$ Abb. 1). Kombiniert wird diese direkte Bypassoperation mit einer indirekten Bypassoperation, z. B. im Rahmen einer Encephalomysynangiosis, bei der das Aussprossen von Gefäßen aus dem Muskel erwartet wird.

Diagnostisch sind die konventionelle zerebrale Angiografie, die Sichtbarmachung der hämodynamischen Situation mittels MR-Perfusion, SPECT oder PET und der Ausschluss von Differenzialdiagnosen wichtig.

Die konservative Behandlung ist ebenso wenig erforscht wie die operative [10]. Unter Experten bestehen unterschiedli- che Meinungen zur Thrombozytenantiaggregation und zur Behandlung von Bewegungsstörungen und Kopfschmerzen [11]. Der vorliegende Fall illustriert den besonderen diagnostischen Stellenwert der Duplex- und Dopplersonografie.

\section{Fallbeschreibung}

Die geplante elektive ambulante Vorstellung der 16-jährigen Maler- und Lackierer-Auszubildenden erfolgte in unserer Duplexambulanz, nachdem bei ihrer Mutter eine idiopathische Moyamoya-Erkrankung von uns diagnostiziert worden war. Wir hatten den Eltern zwar die Seltenheit eines familiären Auftretens bei nichtasiatischen Patienten erläutert, dennoch zur Beruhigung eine Familienuntersuchung angeboten. Bereits im Vorjahr hatte der Bruder der Patientin eine Duplexsonografie von uns mit unauffälligem Befund erhalten, sodass wir eine Moyamoya-Angiopathie bei ihm als äußerst unwahrscheinlich erachteten. Nun hatte die Familie das Angebot für die 16-jährige Tochter in Anspruch genommen.

Bei der Vorstellung vor der Duplexsonografie berichtete die Familie, just seit 5 Tagen könne die Tochter schlechter laufen. Eine Beinschwäche links sei aufgetreten. In der klinisch-neurologischen Untersuchung imponierte ein Absinken im Beinhalteversuch links mit deutlicher linksseitiger Fußheberparese. Duplexsonogra- 

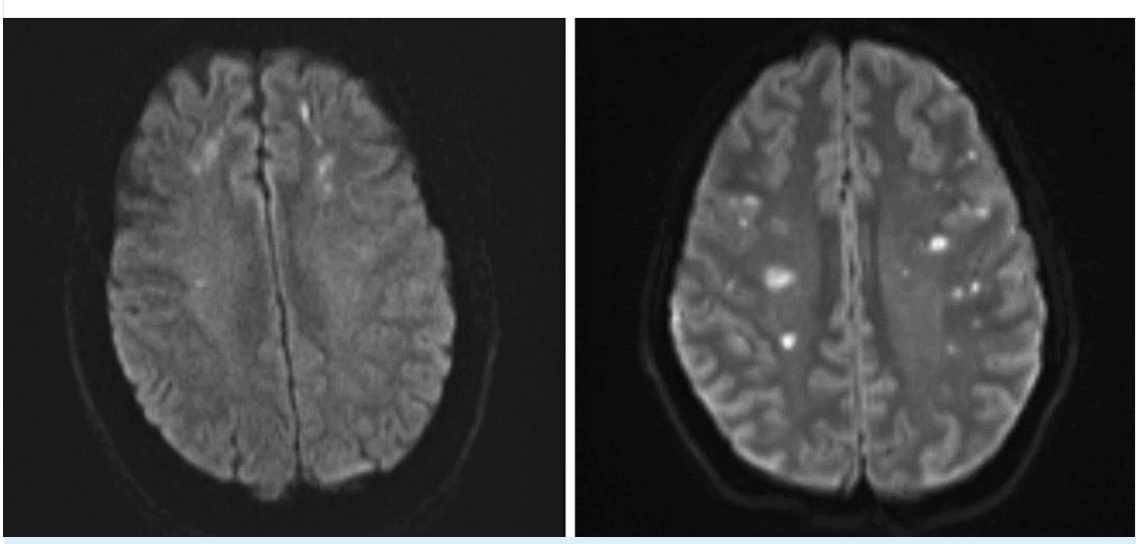

Abb.2 MR-Diffusionswichtung bei Moyamoya-Angiopathie: hämodynamisches oder embolisches Muster?

fisch zeigte sich dann eine hochgradige Stenose mit Musical Murmurs im M1-Segment der A. cerebri media rechts sowie eine Stenose im C1-Segment der A. carotis interna beidseits (ACI links 112/45 cm/s, ACI rechts $110 / 56 \mathrm{~cm} / \mathrm{s}$ ).

Die daraufhin ergänzte spezielle Dopplersonografie zur Messung der Vasomotorenreserve wies bei Ableitung im Mediahauptstamm in $56 \mathrm{~mm}$ Tiefe rechts und $55 \mathrm{~mm}$ Tiefe links eine aufgehobene Vasomotorenreserve im Mediahauptstamm rechts mit $0 \% / \mathrm{mmHg}$ und eine eingeschränkte Vasomotorenreserve links mit $1,4 \% / \mathrm{mmHg}$ nach. Im M2-Segment fand sich in $39 \mathrm{~mm}$ Tiefe rechts und in $41 \mathrm{~mm}$ Tiefe links ebenfalls beidseitig eine aufgehobene Vasomotorenreserve, rechts mit $1,3 \% / \mathrm{mmHg}$, links mit $1,9 \% / \mathrm{mmHg}$. Die spezielle Dopplersonografie zur Emboliedetektion konnte noch am selben Tag 6 HITS (high intensity transient signals) über der rechten Media in 30 Minuten detektieren. Es erfolgte die notfallmäßige Aufnahme der Patientin auf unserer Stroke Unit. Eine Thrombozytenantiaggregation mit ASS 100 und Clopidogrel 75 wurde begonnen.

Die MRT des Hirnschädels zeigte einige embolisch wirkende Diffusionsstörungen im Mediastromgebiet rechts sowie einen Anteriorinfarkt rechts. In der TOF-Angiografie fand sich eine höchstgradige Stenose, DD-Verschluss im M1-Abschnitt der A. cerebri media rechts mit Rarefizierung der peripheren Äste. Außerdem fand sich ein vermindertes Flusssignal im A1-Abschnitt der A. cerebri anterior beidseits.

Die MR-Hirnperfusionsmessung wies allerdings eine akzentuierte Perfusionsminderung vornehmlich im Anteriorstromgebiet mehr als im Mediastromgebiet in der Time-to-Peak-Messung und in der MeanTransit-Time auf. Konventionell angiografisch fanden sich kollateralisierte proxi- male Anteriorverschlüsse beidseits und nur eine Taillierung der C1-Segmente und der Media beidseits.

Die initial duplexsonografisch vermutete hochgradige C1- und M1-Stenose rechts, die auch in der MRT vermutet wurde, stellte sich in dieser Angiografie am 6. Tag der stationären Aufnahme unter ASS sowie Clopidrogrel-Einnahme nicht mehr dar. Korrespondierend dazu war auch die Duplexsonografie im M1-Segment gebessert. Es wurde lediglich eine mittelgradige Stenose der Media rechts mit niederfrequenten Strömungsanteilen (Vmax 207/ $109 \mathrm{~cm} / \mathrm{s}$ ) gesehen. Die hochgradigen Stenosehinweise mit Musical Murmurs wurden nicht mehr nachgewiesen. In der ausführlichen Zusatzdiagnostik inklusive TCD mit Bubbles-Test, transösophagealer Echokardiografie, 48-Stunden-EKG und Thrombophiliediagnostik waren bis auf eine heterozygote Faktor-V-Leiden-Mutation keine Auffälligkeiten zu finden. Auch die ausführliche ätiologische Zusatzdiagnostik und Anamnese inklusive Liquordiagnostik und laborchemischem Vaskulitisscreening sowie allgemeinmedizinisch/ dermatologischer Untersuchung ergaben ebenso wie bei der Mutter keine Ursachen für ein sekundäres Moyamoya-Syndrom. Bei leichtgradiger Beinparese mit deutlicher Fußheberparese folgte eine stationäre Rehabilitationsbehandlung.

Unter der Hypothese einer embolischen Genese der initial nachweisbaren M1-Stenose rechts und der embolisch wirkenden Mediainfarkte rechts bei allerdings angiografisch und duplexsonografisch höchstgradigen Anteriorstenosen nahmen wir Abstand von einer revaskularisierenden Operation zum Mediastromgebiet und planten anstatt dessen eine 15-O-Wasser-PET-Untersuchung, um die hämodynamische Situation im Anteriorstromgebiet weiter aufzuklären. Eine revaskulari- sierende Operation zum Anteriorstromgebiet mittels $A$. temporalis superficalis anterior (STA-ACA)-Operation ist geplant.

\section{Diskussion \\ $\nabla$}

Der vorliegende Fall verdeutlicht den besonderen Stellenwert von Duplexsonografie und spezieller Dopplersonografie in Zusammenschau mit konventioneller Angiografie und MRT. Interessanterweise stellte sich zunächst duplexsonografisch und MR-tomografisch in der TOF-MRA die Media rechts als hochgradig stenosiert dar, allerdings waren schon die vielen HITS in der Emboliedetektion Hinweis auf eine embolische Genese der Infarkte. Diskrepant zur TOF-MRA und Duplexsonografie zeigte sich dann auch in der zeitversetzt durchgeführten konventionellen Angiografie unter ASS und Clopidogrel nur eine leicht- bis mittelgradige M1-Stenose rechts. Entsprechend war die spezielle Dopplersonografie zur Emboliedetektion normalisiert und duplexsonografisch nur noch eine mittelgradige Stenose nachweisbar.

Die Kombination der verschiedenen zusatzdiagnostischen Methoden erlaubte uns, von einem embolischen Geschehen mit embolischer Mediastenose auf der Grundlage einer vorbestehenden mittelgradigen Mediastenose auszugehen. Die Unterscheidung in hämodynamische und embolische Ischämien ist wegen der Frage einer revaskularisierenden Operation bei der Moyamoya-Erkrankung dringend erforderlich ( Abb.2). Wir verzichteten auf eine revaskularisierende Operation zum Mediastromgebiet, da in den hämodynamischen Zusatzuntersuchungen sich trotz auch im Verlauf pathologischer Dopplersonografie zur Vasomotorenreserve die Durchblutungssituation im Mediastromgebiet als ausreichend darstellte. Zum Anteriorstromgebiet gehen wir allerdings von einer deutlichen hämodynamischen Kompromittierung aus und planen eine revaskularisierende Operation mittels STA-ACA-Bypass beidseits.

Der vorliegende besondere Fall verdeutlicht nicht nur den Stellenwert der - oft vernachlässigten - nicht invasiven Duplexsonografie in der Diagnostik der Moyamoya-Angiopathie [12-14], sondern auch die Wichtigkeit einer Thrombozytenantiaggregation bei dieser Erkrankung [11]. Bei Experten zur MoyamoyaAngiopathie ist die Thrombozytenantiaggregation umstritten, insbesondere wird traditionell von japanischen Kollegen aus 

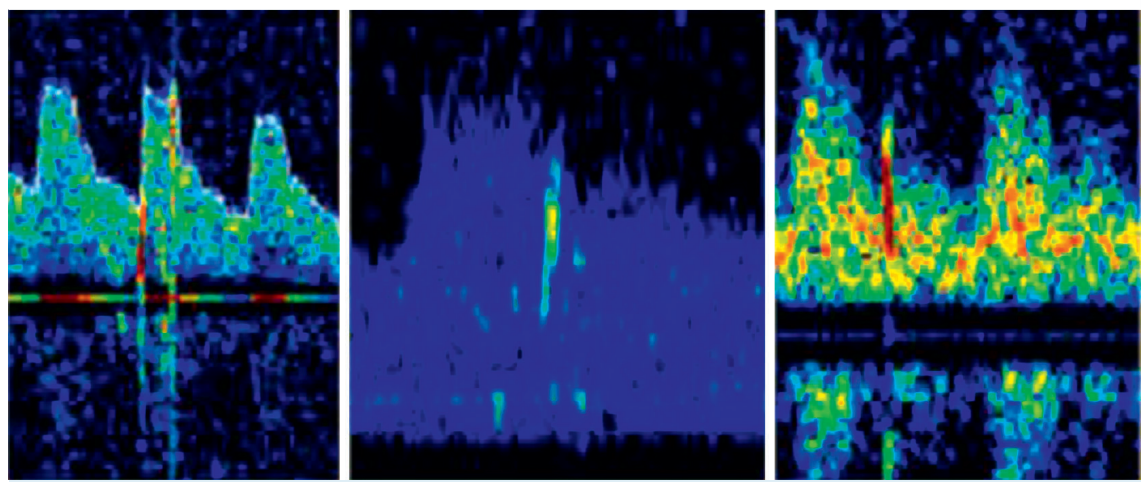

Abb.3 Transkranielle Dopplersonografie zur Emboliedetektion. Aus [22] mit freundlicher Genehmigung.

Sorge vor zerebralen Blutungen vor dieser zurückgescheut [11].

Bereits in den 1990er- und 2000er-Jahren wurde die Emboliedetektion als Methode zur Detektion von embolischen Stenosen wissenschaftlich untersucht ( Abb.3) $[15,16]$. Sowohl höhergradige Stenosen als auch mittel- bis leichtgradigere Verengungen waren als HITS-Clustern assoziiert beschrieben $[16,17]$.

Jüngst wurden mikroembolische Signale bei Moyamoya-Angiopathie systematisch untersucht [18]. Bei 20\% der 55 asiatischen Patienten mit Moyamoya-Angiopathie traten mikroembolische Signale auf und diese waren mit ischämischen Ereignissen in den letzten 3 Monaten assoziiert. Die Hazard Ratio für einen weiteren

\section{Was lernt man daraus?}

Obwohl klinisch und wissenschaftlich eine hämodynamische Genese der flüchtigen und manifesten Ischämien bei der Moyamoya-Angiopathie im Vordergrund steht, muss berücksichtigt werden, dass bei Stenosen immer auch eine embolische Pathologie möglich ist und dies geprüft werden muss. Dies verdeutlicht auch das Erfordernis einer Thrombocytenantiaggregation nach einer revaskularisierenden Operation. Gerade die heute nur noch selten praktizierte - da zeitaufwendige und personalintensive "alte Methode“ - der speziellen Dopplersonografie zur Emboliedetektion ist ein wertvolles diagnostisches Instrument bei der Frage nach einer embolischen Genese von ischämischen MR-tomografischen Auffälligkeiten [19-21]. Die Wechselwirkung zwischen schlechter hämodynamischer Situation und Embolien (reduziertem wash-out von Embolien) muss berücksichtigt werden.
Schlaganfall bei Nachweis der auffälligen Emboliedetektion lag bei 10,61 (95\%-KI $1,66-67,70)[18]$.

Interessenkonflikt: Der Autor gibt an, dass kein Interessenkonflikt besteht.

\section{Literatur}

1 Kramer M, Berlit P. [Moyamoya disease - a rare vasculopathy in Europeans]. Fortschritte der Neurologie-Psychiatrie 2010; 78: 542 - 550; quiz 551

2 Houkin K, Ito M, Sugiyama $T$ et al. Review of past research and current concepts on the etiology of moyamoya disease. Neurologia medico-chirurgica 2012; 52: 267 - 277

3 Wakai K, Tamakoshi A, Ikezaki Ket al. Epidemiological features of moyamoya disease in Japan: findings from a nationwide survey. Clinical neurology and neurosurgery 1997; 99 (Suppl. 02): S1-5

4 Baba T, Houkin K, Kuroda S. Novel epidemiological features of moyamoya disease. Journal of neurology, neurosurgery, and psychiatry 2008; 79: $900-904$

5 Achrol AS, Guzman R, Lee $M$ et al. Pathophysiology and genetic factors in moyamoya disease. Neurosurgical focus 2009; 26: E4

6 Fujimura M, Sonobe S, Nishijima Y et al. Genetics and Biomarkers of Moyamoya Disease: Significance of RNF213 as a Susceptibility Gene. Journal of stroke 2014; 16: 65 72

7 Liu W, Senevirathna ST, Hitomi T et al. Genomewide association study identifies no major founder variant in Caucasian moyamoya disease. Journal of genetics 2013; 92: 605-609

8 Kraemer $M$, Heienbrok W, Berlit P. Moyamoya disease in Europeans. Stroke; a journal of cerebral circulation 2008; 39: 3193-3200

9 Guzman R, Lee M, Achrol A et al. Clinical outcome after 450 revascularization procedures for moyamoya disease. Clinical article. Journal of neurosurgery 2009; 111: 927 935

10 Kronenburg A, Braun KP, van der Zwan A et al. Recent advances in moyamoya disease: pathophysiology and treatment. Current neurology and neuroscience reports 2014; 14: 423

11 Kraemer $M$, Berlit $P$, Diesner $F$ et al. What is the expert's option on antiplatelet therapy in moyamoya disease? Results of a worldwide Survey. Eur J Neurol 2012; 19: 163 167

12 Kraemer M, Schuknecht B, Jetzer AK et al. Postoperative changes in the superficial temporal artery and the external carotid artery duplex sonography after extra-intracranial bypass surgery in European Moyamoya disease. Clinical neurology and neurosurgery 2012; 114: 930 -934

13 Perren F, Meairs S, Schmiedek P et al. Power Doppler evaluation of revascularization in childhood moyamoya. Neurology 2005; 64: 558-560

14 Perren F, Horn P, Vajkoczy $P$ et al. Power Doppler imaging in detection of surgically induced indirect neoangiogenesis in adult moyamoya disease. Journal of neurosurgery 2005; 103: $869-872$

15 Segura T, Serena J, Molins A et al. Clusters of microembolic signals: a new form of cerebral microembolism presentation in a patient with middle cerebral artery stenosis. Stroke; a journal of cerebral circulation 1998; 29: $722-724$

16 Alexandrov AV, Demchuk AM, Felberg RA et al. Intracranial clot dissolution is associated with embolic signals on transcranial Doppler. Journal of neuroimaging: official journal of the American Society of Neuroimaging 2000; 10: $27-32$

17 Diehl RR, Samii C, Diehl A. Dynamics and embolic activity of symptomatic intra-cranial cerebral artery stenoses. Acta Neurol Scand 2002; 106: $173-181$

18 Chen J, Duan L, Xu WH et al. Microembolic signals predict cerebral ischaemic events in patients with moyamoya disease. Eur J Neurol 2014; 21: 785-790

19 Sliwka U, Diehl RR, Noth J. [The significance of so-called "high-intensity transient signals" (HITS) during transcranial Doppler sonography]. Deutsche medizinische Wochenschrift 1995; 120: 1667 - 1670

20 Sliwka U, Job FP, Wissuwa D et al. Occurrence of transcranial Doppler high-intensity transient signals in patients with potential cardiac sources of embolism. A prospective study. Stroke; a journal of cerebral circulation 1995; 26: $2067-2070$

21 Hennerici MG. High intensity transcranial signals (HITS): a questionable 'jackpot' for the prediction of stroke risk. The Journal of heart valve disease 1994; 3: 124-125

22 Diehl RR, Berlit P. Funktionelle Dopplersonographie in der Neurologie. Berlin, Heidelberg: Springer; 1996

\section{Duplex and Doppler Sonography in Moyamoya Angiopathy}

Autor

M. Krämer

Institut

Neurologische Klinik des Alfried Krupp von Bohlen und Halbach Krankenhauses, Essen

\section{Korrespondenzadresse}

\section{Priv.-Doz. Dr. Markus Krämer}

Neurologische Klinik, Alfried Krupp von Bohlen und Halbach Krankenhaus gGmbH

Alfried-Krupp-Straße 21

45117 Essen

Markus.kraemer@krupp-krankenhaus.de

\section{Bibliografie}

DOI http://dx.doi.org/10.1055/s-0034-1387512

Online-Publikation: 8.1.2015

Klin Neurophysiol 2015; 46: 47-49

(c) Georg Thieme Verlag KG

Stuttgart · New York

ISSN 1434-0275 